\title{
On some properties of the quadrilateral.
}

By R. E. Allardice, M.A.

§1. In a triangle $\mathrm{ABC}$ (fig. 37 ), $\mathrm{BE}$ is made equal to $\mathrm{CF}$; to find the locus of the middle point of EF.

Take $\mathrm{K}$ the middle point of $\mathrm{BC}$ and $\mathrm{P}$ the middle point of EF, then $\mathrm{PK}$ is the locus required. For if $\mathrm{E}^{\prime}$ and $\mathrm{F}^{\prime}$ are the middle points of $\mathrm{BE}$ and $\mathrm{CF}$, the middle point of $\mathrm{E}^{\prime} \mathrm{F}^{\prime}$ will lie in $\mathrm{PK}$ (namely, at the middle point of $\mathrm{PK}$ ); and again if $\mathrm{BE}^{\prime}$ and $\mathrm{CF}^{\prime}$ are bisected in $\mathrm{E}^{\prime \prime}$ and $\mathrm{F}^{\prime \prime}$, the middle point of $\mathrm{E}^{\prime \prime} \mathrm{F}^{\prime \prime}$ will lie in $\mathrm{PK}$ (namely, at the middle point of $\mathrm{KR}$ ); and so on. At any stage we may double the parts cut off from BA and CA instead of bisecting them. Hence the locus required is such that any part of it, however small, contains an infinite number of collinear points; and hence the locus is a straight line.

§2. The proof of the above paragraph obviously holds good if $\mathrm{BE}$ and $\mathrm{CF}$, instead of being taken equal, are taken in a constant ratio.

Hence the proposition may be stated as a property of the quad rilateral, as follows:-

If the points $P$ and $Q$ divide the sides $A B$ and $D C$ of a quadrilateral $A B C D$ in the same (variable) ratio, the locus of the middle point of $P Q$ is a straight line.

\section{§ 3. Generalisation.}

If $L$ and $M$ (fig. 38) divide the sides $A B$ and $D C$ of a quadrilateral $A B C D$ in the same (variable) ratio $l: m$, then the locus of a point $P$ that divides $L M$ in a given ratio is a straight line.

For this is true, by last paragraph, if we take the ratio LP:LM to be $1: 2$; and hence also if we take it to be $1: 4$ or $1: 8$ or $3: 8$; or, in general, if we take it to be $m: 2^{n}$; and hence it must be true generally.

Note.-In this way the whole plane may be divided into a number of quadrilaterals whose sides are proportional (but which are not similar).

\section{§4. Second Method of Proof.}

Lemma.-Let ABC, DEF (fig. 39) be two straight lines and DA, 
EB, FC, be perpendicular to $A C$; and let further $A^{\prime} B^{\prime} C^{\prime}$ (fig. 40 ) be a straight line and $D^{\prime} A^{\prime}, E^{\prime} B^{\prime}, F^{\prime} C^{\prime}$ be perpendiculars to $A^{\prime} C^{\prime}$, equal respectively to $D A, E B, F C$; then if $A B: B C=A^{\prime} B^{\prime}: B^{\prime} C^{\prime}$, the points $D^{\prime}, E^{\prime}, F^{\prime}$, are collinear ; and conversely.

The proof of this lemma comes at once on drawing through $\mathrm{D}$ and $\mathrm{D}^{\prime}$ lines parallel respectively to $\mathrm{AC}$ and $\mathrm{A}^{\prime} \mathrm{C}^{\prime}$.

[This lemma is obviously connected with a property of the simlpest kind of homogeneous strain.]

Now take (fig. 41) BF:FD =CG:GE. Bisect $\mathrm{BC}$ and $\mathrm{DE}$ in $\mathrm{P}$ and $Q$, and join $P Q$. Draw perpendiculars $B H, C H^{\prime}$, etc., to $P Q$. Then, obviously, $\mathrm{BH}=\mathrm{CH}^{\prime}, \mathrm{DL}=\mathrm{EL}^{\prime}$; and $\mathrm{HK}: \mathrm{KL}=\mathrm{H}^{\prime} \mathrm{K}^{\prime}: \mathrm{K}^{\prime} \mathrm{L}^{\prime}$; and hence $\mathrm{FK}=\mathrm{GK}^{\prime}$ and $\mathrm{FR}=\mathrm{RG}$.

The more general theorem of $(\S 3)$ may also be proved in this way.

\section{§5. Third Method of Proof.}

As neither of the preceding proofs is exactly Euclidian in character, it may be as well to add the following proof.

Let $\mathrm{ABC}$ (fig. 42) be any triangle.

Make $\mathrm{AC}^{\prime}=\mathrm{AC} ; \mathrm{AB}^{\prime}=\mathrm{AB}$.

Then $\mathrm{BB}^{\prime}$ is parallel to $\mathrm{CC}^{\prime}$, and $\mathrm{P}, \mathrm{Q}, \mathrm{R}, \mathrm{S}$, the middle points of $\mathrm{BC}, \mathrm{BC}^{\prime}, \mathrm{B}^{\prime} \mathrm{C}, \mathrm{B}^{\prime} \mathrm{C}^{\prime}$, are collinear.

Now $B Q=C R=\frac{1}{2}(B A+A C)$.

If we make $\mathrm{BD}=\mathrm{CE}$, we have still $\mathrm{DQ}=\mathrm{ER}=\frac{1}{2}(\mathrm{DA}+\mathrm{AE})$; and hence $R Q$ passes through $P^{\prime}$, the middle point of $\mathrm{DE}$.

The more general theorem, in which $\mathrm{BD}: \mathrm{CE}$ is a constant ratio, may be proved in much the same way.

$\S 6$. By means of the proposition of the preceding paragraphs, a simple proof may be given of the following well-known theorem * -

The middle points of the diagonals of a complete quadrilateral are concurrent. (Fig. 43).

Make BHDK a parallelogram.

Then $\mathrm{AH} / \mathrm{HE}=(\mathrm{AH} / \mathrm{HB})(\mathrm{HB} / \mathrm{HE})$

$$
\begin{aligned}
& =(\mathrm{BK} / \mathrm{KF})(\mathrm{KC} / \mathrm{KB}) \\
& =\mathrm{KC} / \mathrm{KF} .
\end{aligned}
$$

Hence the middle points of $\mathrm{HK}, \mathrm{AC}$ and $\mathrm{EF}$ are collinear.

* Numerous proofs of this theorem have been given, one of the simplest being that contained in Taylor's Ancient and Modern Geometry of Conics, \$107. For some account of the history of the theorem, see the last paper in this volume of the Proceed. ings, by Dr J. S Mackuy. 
$\S 7$. The following proof, by means of co-ordinates, of the general theorem of $(\S 3)$ is so simple, that it may be worth while giving it here.

Put (fig. 38)

$$
\begin{aligned}
\mathrm{AL}: \mathrm{LB} & =\mathrm{DM}: \mathrm{MC}=\lambda: \mu ; \\
\mathrm{AR}: \mathrm{RD} & =\mathrm{LP}: \mathrm{PM}=\mathrm{BS}: \mathrm{SC}=p: q .
\end{aligned}
$$

Let the co-ordinates of $\mathrm{R}, \mathrm{P}, \mathrm{Q}$, be $\left(\xi_{1}, \eta_{1}\right),\left(\xi_{2}, \eta_{2}\right),\left(\xi_{3}, \eta_{3}\right)$; the co-ordinates of $\mathrm{A}$ be $\left(x_{1}, y_{1}\right)$, etc.

Then $\xi_{1}=\left(q x_{1}+p x_{4}\right) /(p+q)$.

$$
\begin{aligned}
\xi_{2} & =\left\{p\left(\lambda x_{3}+\mu x_{4}\right) /(\lambda+\mu)+q\left(\lambda x_{2}+\mu x_{1}\right) /(\lambda+\mu)\right\} /(p+q) \\
& =\left\{p\left(\lambda x_{3}+\mu x_{4}\right)+q\left(\lambda x_{2}+\mu x_{1}\right)\right\} /(\lambda+\mu)(p+q) . \\
\xi_{3} & =\left(p x_{3}+q x_{2}\right) /(p+q) .
\end{aligned}
$$

Now we may easily show that if we put

$$
\begin{aligned}
& \mathbf{P}=\left\{p\left(x_{4}-x_{3}\right)+q\left(x_{1}-x_{2}\right)\right\} /(\lambda+\mu)(p+q), \\
& \mathbf{Q}=\left\{p\left(y_{4}-y_{3}\right)+q\left(y_{1}-y_{2}\right)\right\} /(\lambda+\mu)(p+q),
\end{aligned}
$$

then

$$
\xi_{2}-\xi_{3}=\mu \mathrm{P} ; \xi_{3}-\xi_{1}=-(\lambda+\mu) \mathrm{P} ; \xi_{1}-\xi_{2}=\lambda \mathrm{P} \text {. }
$$

Hence

$$
\begin{aligned}
& \eta_{1}\left(\xi_{2}-\xi_{3}\right)+\eta_{2}\left(\xi_{3}-\xi_{1}\right)+\eta_{3}\left(\xi_{1}-\xi_{2}\right) \\
& \quad=\eta_{1} \mu \mathrm{P}-\eta_{2}(\lambda+\mu) \mathrm{P}+\eta_{3} \lambda \mathrm{P} \\
& \quad=\mathrm{P}\left\{\lambda\left(\eta_{3}-\eta_{2}\right)+\mu\left(\eta_{1}-\eta_{2}\right)\right\} \\
& \quad=\mathrm{P}\{\lambda(-\mu \mathrm{Q})+\mu(\lambda \mathrm{Q})\} \\
& \quad=\mathrm{PQ}(-\lambda \mu+\lambda \mu)=0 .
\end{aligned}
$$

Hence $\mathrm{R}, \mathrm{P}$ and $\mathrm{S}$ are collinear.

An Apparatus of Professor Tait's was exhibited which gives the same curve as a glissette, either of a byperbola or an ellipse.

Fourth Meeting, 28th February 1890.

R. E. Allardice, Esq., M.A., Vice-President, in the Chair.

On the Moduluses of Elasticity of an Elastic Solid according to Boscovich's Theory.

By Sir William Thomson.

The substance of this paper will be found in the Proceedings of the Royal Society of Edinburgh, Vol. xvi., pp. 693-724; and Thomson's Mathematical and Physical Papers, Vol. iii., Art. xcvii., pp. $395-498$. 